\title{
Primary Tumor Score Based on Tumor Depth and Length Predicts Prognosis in Esophageal Squamous Cell Carcinoma
}

\author{
TAKAAKI ARIGAMI $^{1}$, YASUTO UCHIKADO ${ }^{2}$, ITARU OMOTO ${ }^{2}$, KEN SASAKI $^{2}$, \\ YOSHIAKI KITA ${ }^{2}$, TETSUHIRO OWAKI ${ }^{3}$, SHIGEHIRO YANAGITA ${ }^{2}$, SHINICHIRO MORI ${ }^{2}$, \\ HIROSHI KURAHARA ${ }^{2}$, HIROSHI OKUMURA ${ }^{2}$, KOSEI MAEMURA ${ }^{2}$ and SHOJI NATSUGOE ${ }^{1,2}$ \\ ${ }^{1}$ Department of Onco-biological Surgery, Kagoshima University Graduate \\ School of Medical and Dental Sciences, Kagoshima, Japan; \\ ${ }^{2}$ Department of Digestive Surgery, Breast and Thyroid Surgery, \\ and ${ }^{3}$ Education Center for Doctors in Remote Islands and Rural Areas, \\ Kagoshima University Graduate School of Medical and Dental Sciences, Kagoshima, Japan
}

\begin{abstract}
Aim: To examine the depth of tumor invasion and tumor length and assess the clinical impact of the primary tumor score (PTS), based on a combination of tumor invasion and tumor length, in patients with esophageal squamous cell carcinoma (ESCC). Patients and Methods: A total of 237 patients with ESCC were classified into three PTS groups based on cut-off values for deeper tumor invasion (pT2-T4) and greater tumor length $(\geq 44 \mathrm{~mm})$. A PTS of 2 indicated the presence of both of these abnormalities, 1 indicated one of these abnormalities, and 0 indicated neither abnormality. Results: PTS was significantly positively correlated with depth of tumor invasion, lymph node metastasis, lymphovascular invasion, and stage (all $p<0.001)$. The prognosis differed significantly among the three groups based on PTS $(p<0.0001)$. Multivariate analysis demonstrated that PTS was an independent prognostic factor ( $p=0.0004)$. Conclusion: PTS has a clinical utility as a prognostic predictor in patients with ESCC.
\end{abstract}

Esophageal squamous cell carcinoma (ESCC) is one of the most aggressive malignancies in patients with gastrointestinal cancer and is the seventh leading cause of cancer-specific death in Japan (1). The 5-year survival rates of patients with stage I, II, III, and IV ESCC are 90\%, 45\%, $20 \%$, and $10 \%$, respectively (2). Even patients with

Correspondence to: Takaaki Arigami, MD, Ph.D., Department of Onco-biological Surgery, Kagoshima University Graduate School of Medical and Dental Sciences, 8-35-1 Sakuragaoka, Kagoshima 890-8520, Japan. Tel: +81 992755361, Fax: +81 992657426, e-mail: arigami@m.kufm.kagoshima-u.ac.jp

Key Words: Primary tumor score, depth of tumor invasion, tumor length, prognosis, esophageal squamous cell carcinoma. submucosal invasion have a high potential for lymph node metastasis, and the metastatic rate of lymph nodes in these patients is reported to be $16-62 \%$ (3). The 5 -year diseasespecific survival rate of patients with deep submucosal invasion is $68.6-78.8 \%$ (3). These findings indicate that it is difficult to plan a therapeutic strategy in the clinical management of ESCC. Although induction of adjuvant chemotherapy to prevent disease recurrence after surgery is clinically recommended in patients with pathological stage II or III gastric cancer, the clinical impact of adjuvant chemotherapy in patients with ESCC remains unclear (4). Carcinoembryonic antigen (CEA), squamous cell carcinoma antigen (SCC), and p53 are used as representative blood markers for predicting disease recurrence in the postoperative management of ESCC. However, the sensitivity of these conventional serum markers for predicting disease recurrence in patients with ESCC is 25$40 \%$ (5). Promising predictors of tumor progression and prognosis are awaited in the clinical management of ESCC.

Currently, postoperative patients with ESCC are categorized and staged by pathological examinations based on the criteria of the tumor-node-metastasis (TNM) classification of esophageal carcinoma established by the Union for International Cancer Control (UICC) (6). The TNM classification is based on three clinicopathological factors: the depth of tumor invasion, and the presence of lymph node metastases, and distant metastases. It is impossible to determine TNM stage without information on these three factors. If a new pathological score determined by primary tumor information alone had the same clinical utility as TNM stage, it would be possible to predict disease recurrence and prognosis without requiring information about the presence or absence of lymph node and distant metastases.

In this study, we focused on tumor depth and length and defined a primary tumor score (PTS) based on these two 
primary tumor factors. To date, the clinical significance of PTS determined by tumor depth and length has not been assessed in patients with ESCC.

The aim of the present study was to investigate tumor depth and length in patients with ESCC, and examine the relationship between PTS, clinicopathological factors and long-term outcomes for assessing its clinical utility as a prognostic combined marker.

\section{Patients and Methods}

Patients. Data were retrospectively reviewed from 237 patients (209 men and 28 women; age range $=37-87$ years; average $=65.3$ years) with ESCC who underwent esophagectomy with lymphadenectomy at the Kagoshima University Hospital (Kagoshima, Japan) between January 1998 and December 2012. Patients who had received endoscopic treatments, palliative resection, preoperative chemotherapy, or radiotherapy were excluded. None of the enrolled patients had synchronous or metachronous cancer in other organs. In the present study, patients were classified and staged based on the TNM classification of esophageal carcinoma established by the UICC (6). Table I shows the clinicopathological factors. All patients were followed-up every 3 to 6 months after surgery at the Kagoshima University Hospital with regular clinical diagnostic examinations, including tumor marker (CEA, SCC, and p53) studies, radiography, ultrasonography, and computed tomography. The median follow-up period was 26 months (range=1-182 months).

The Ethics Committee of the Kagoshima University approved this observational study (approval number 180058).

Pathological examination for tumor depth and length. The depth of tumor invasion was determined by the TNM classification of esophageal carcinoma. According to this classification, tumors are pathologically grouped into four divisions: pT1, pT2, pT3, and pT4. Tumor length was calculated based on the Japanese Classification of Esophageal Cancer (11th edition) (7). The resected esophagus was opened along the longitudinal line on the side opposite to the lesion. Tumor length was defined as the greatest longitudinal dimension.

The primary end-point was overall survival. Overall survival was defined as the period between surgery and death. All deaths, including those from other diseases, were considered to be events. Data for patients without an event were censored at the date of the final observation.

Statistical analysis. The chi-square test and Fisher's exact test were used to evaluate the relationships between tumor depth and categorical clinicopathological factors. Differences in the relationships between tumor length and clinicopathological features were assessed by the Wilcoxon rank sum test. Receiver operating characteristic (ROC) curves were constructed and the area under the curve (AUC) was calculated to estimate the predictive ability of tumor length for distinguishing patients with stage I from those with stage II or III cancer. The relationship between PTS and clinicopathological factors was evaluated by the chi-square test or Fisher's exact test. Survival curves were generated by the KaplanMeier method, and differences in survival were examined by the log-rank test. Prognostic factors were assessed by univariate and multivariate analyses (Cox proportional hazards regression model). All statistical calculations were performed with SAS statistical
Table I. Clinicopathological factors of 237 patients with esophageal squamous cell carcinoma.

\begin{tabular}{lc}
\hline Characteristic & Value \\
\hline Gender, n (\%) & \\
Male & $209(88.2 \%)$ \\
Female & $28(11.8 \%)$ \\
Mean age (range), years & $65.3(37-87)$ \\
Tumor location, $\mathrm{n}(\%)$ & \\
Upper & $42(17.7 \%)$ \\
Middle & $115(48.5 \%)$ \\
Lower & $80(33.8 \%)$ \\
Mean tumor length(range), mm & $48.4(4-155)$ \\
Depth of tumor invasion, $\mathrm{n}(\%)$ & \\
pT1 & $119(50.2 \%)$ \\
pT2 & $25(10.5 \%)$ \\
pT3 & $82(34.6 \%)$ \\
pT4 & $11(4.6 \%)$ \\
Lymph node metastasis, $\mathrm{n}(\%)$ & \\
Negative & $113(47.7 \%)$ \\
Positive & $124(52.3 \%)$ \\
Stage, $\mathrm{n}(\%)$ & \\
I & $86(36.3 \%)$ \\
II & $69(29.1 \%)$ \\
III & $82(34.6 \%)$ \\
Venous invasion, $\mathrm{n}(\%)$ & \\
Negative & $99(41.8 \%)$ \\
Positive & $138(58.2 \%)$ \\
Lymphatic invasion, $\mathrm{n}(\%)$ & \\
Negative & $94(39.7 \%)$ \\
Positive & $143(60.3 \%)$ \\
\hline
\end{tabular}

software (SAS Institute Inc., Cary, N.C., USA). A p-value of less than 0.05 was considered to represent a statistically significant difference.

\section{Results}

Correlation between clinicopathological factors and tumor depth or length. One hundred and nineteen, 25, 82, and 11 patients had pT1, pT2, pT3, and pT4 tumors, respectively. The relationships between tumor depth and clinicopathological factors are shown in Table II. Tumor depth was significantly positively correlated with lymph node metastasis, stage, venous invasion, and lymphatic invasion (all $p<0.0001$ ).

Tumor length ranged from 4 to $155 \mathrm{~mm}$. The mean tumor length $( \pm$ SD) was $48.4 \pm 25.5 \mathrm{~mm}$. The tumor length was significantly correlated with the depth of tumor invasion (Figure $1 \mathrm{~A} ; p<0.0001)$. Tumor length was significantly greater in patients with lymph node metastases $(n=124)$ than in those without $(n=113)$ (Figure $1 \mathrm{~B} ; p=0.0066)$. Tumor length was significantly correlated with the presence venous invasion (Figure 1C; $p=0.0025$ ), but was not significantly correlated with lymphatic invasion (Figure 1D; $p=0.0842$ ). 
Table II. Correlation between clinicopathological factors and tumor depth.

\begin{tabular}{|c|c|c|c|c|c|}
\hline \multirow[t]{2}{*}{ Factor } & \multicolumn{4}{|c|}{ Depth of tumor invasion, $\mathrm{n}(\%)$} & \multirow[b]{2}{*}{$p$-Value } \\
\hline & pT1 (n=119) & $\mathrm{pT} 2(\mathrm{n}=25)$ & pT3 $(n=82)$ & pT4 $(n=11)$ & \\
\hline \multicolumn{6}{|l|}{ Gender } \\
\hline Male & $103(86.6)$ & $23(92.0)$ & $74(90.2)$ & $9(81.8)$ & 0.7021 \\
\hline Female & $16(13.4)$ & $2(8.0)$ & $8(9.8)$ & $2(18.2)$ & \\
\hline \multicolumn{6}{|l|}{ Age } \\
\hline$<70$ Years & $93(78.2)$ & $16(64.0)$ & $61(74.4)$ & $8(72.7)$ & 0.5134 \\
\hline$\geq 70$ Years & $26(21.8)$ & $9(36.0)$ & $21(25.6)$ & $3(27.3)$ & \\
\hline \multicolumn{6}{|c|}{ Lymph node metastasis } \\
\hline Negative & $77(64.7)$ & $9(36.0)$ & $24(29.3)$ & $3(27.3)$ & $<0.0001$ \\
\hline Positive & $42(35.3)$ & $16(64.0)$ & $58(70.7)$ & $8(72.7)$ & \\
\hline \multicolumn{6}{|l|}{ Stage } \\
\hline I & $77(64.7)$ & $9(36.0)$ & $0(0.0)$ & $0(0.0)$ & $<0.0001$ \\
\hline II & $36(30.3)$ & $9(36.0)$ & $24(29.3)$ & $0(0.0)$ & \\
\hline III & $6(5.0)$ & $7(28.0)$ & $58(70.7)$ & $11(100.0)$ & \\
\hline \multicolumn{6}{|c|}{ Venous invasion } \\
\hline Negative & $77(64.7)$ & $6(24.0)$ & $14(17.1)$ & $2(18.2)$ & $<0.0001$ \\
\hline Positive & $42(35.3)$ & $19(76.0)$ & $68(82.9)$ & $9(81.8)$ & \\
\hline \multicolumn{6}{|c|}{ Lymphatic invasion } \\
\hline Negative & $68(57.1)$ & $7(28.0)$ & $15(18.3)$ & $4(36.4)$ & $<0.0001$ \\
\hline Positive & $51(42.9)$ & $18(72.0)$ & $67(81.7)$ & $7(63.6)$ & \\
\hline
\end{tabular}

There was a significant positive relationship between tumor length and stage (Figure 1E; $p<0.0001$ ).

ROC analysis was used to assess the predictive power of tumor length for discriminating patients with early cancer from those with advanced cancer. The cutoff value of tumor length was set at $44.0 \mathrm{~mm}$ by ROC analysis (Figure 1F). The AUC, sensitivity, and specificity were $0.679,0.656$, and 0.640 , respectively. Based on the cutoff value of tumor length, patients were divided into two groups with tumor length of $\geq 44 \mathrm{~mm}(\mathrm{n}=130)$ and $<44 \mathrm{~mm}(\mathrm{n}=107)$. This binary classification was used in subsequent analyses.

Definition of PTS. PTS was assigned based on tumor depth and length. PTS was defined as 2 for presence of both deeper tumor invasion (pT2-T4) and greater tumor length ( $\geq 44 \mathrm{~mm}$ ); 1 when either deeper tumor invasion or greater tumor length were found; or 0 when neither deeper tumor invasion nor greater tumor length were found.

Seventy patients $(29.5 \%)$ had a PTS of 0,86 patients $(36.3 \%)$ had a PTS of 1 , and 81 patients $(34.2 \%)$ had a PTS of 2 .

Correlation between clinicopathological factors and PTS. PTS was significantly positively associated with depth of tumor invasion, lymph node metastasis, stage, venous invasion, and lymphatic invasion $(p<0.0001, p<0.0001, p<0.0001$, $p<0.0001$, and $p=0.0001$, respectively) (Table III).
Table III. Correlation between clinicopathological factors and primary tumor score (PTS).

\begin{tabular}{|c|c|c|c|c|}
\hline \multirow[t]{2}{*}{ Factor } & \multicolumn{3}{|c|}{ PTS, n (\%) } & \multirow[t]{2}{*}{$p$-Value } \\
\hline & $0(n=70)$ & $1(n=86)$ & $2(n=81)$ & \\
\hline \multicolumn{5}{|l|}{ Gender } \\
\hline Male & $59(84.3)$ & 78 (90.7) & $72(88.9)$ & \multirow[t]{2}{*}{0.4536} \\
\hline Female & $11(15.7)$ & $8(9.3)$ & $9(11.1)$ & \\
\hline \multicolumn{5}{|l|}{ Age } \\
\hline$<70$ Years & $53(75.7)$ & 65 (75.6) & $60(74.1)$ & \multirow[t]{2}{*}{0.9654} \\
\hline$\geq 70$ Years & $17(24.3)$ & $21(24.4)$ & $21(25.9)$ & \\
\hline \multicolumn{5}{|c|}{$\begin{array}{l}\text { Depth of } \\
\text { tumor invasion }\end{array}$} \\
\hline pT1 & $70(100.0)$ & $49(57.0)$ & $0(0.0)$ & \multirow[t]{4}{*}{$<0.0001$} \\
\hline pT2 & $0(0.0)$ & $14(16.3)$ & $11(13.6)$ & \\
\hline pT3 & $0(0.0)$ & $21(24.4)$ & $61(75.3)$ & \\
\hline pT4 & $0(0.0)$ & $2(2.3)$ & $9(11.1)$ & \\
\hline \multicolumn{5}{|c|}{ Lymph node metastasis } \\
\hline Negative & $49(70.0)$ & $41(47.7)$ & $23(28.4)$ & \multirow[t]{2}{*}{$<0.0001$} \\
\hline Positive & $21(30.0)$ & $45(52.3)$ & $58(71.6)$ & \\
\hline \multicolumn{5}{|l|}{ Stage } \\
\hline I & $49(70.0)$ & $34(39.5)$ & $3(3.7)$ & \multirow[t]{3}{*}{$<0.0001$} \\
\hline II & $18(25.7)$ & $30(34.9)$ & $21(25.9)$ & \\
\hline III & $3(4.3)$ & $22(25.6)$ & $57(70.4)$ & \\
\hline \multicolumn{5}{|c|}{ Venous invasion } \\
\hline Negative & 43 (61.4) & $44(51.2)$ & $12(14.8)$ & \multirow[t]{2}{*}{$<0.0001$} \\
\hline Positive & $27(38.6)$ & $42(48.8)$ & $69(85.2)$ & \\
\hline \multicolumn{5}{|c|}{ Lymphatic invasion } \\
\hline Negative & $39(55.7)$ & $37(43.0)$ & $18(22.2)$ & \multirow[t]{2}{*}{0.0001} \\
\hline Positive & $31(44.3)$ & $49(57.0)$ & $63(77.8)$ & \\
\hline
\end{tabular}



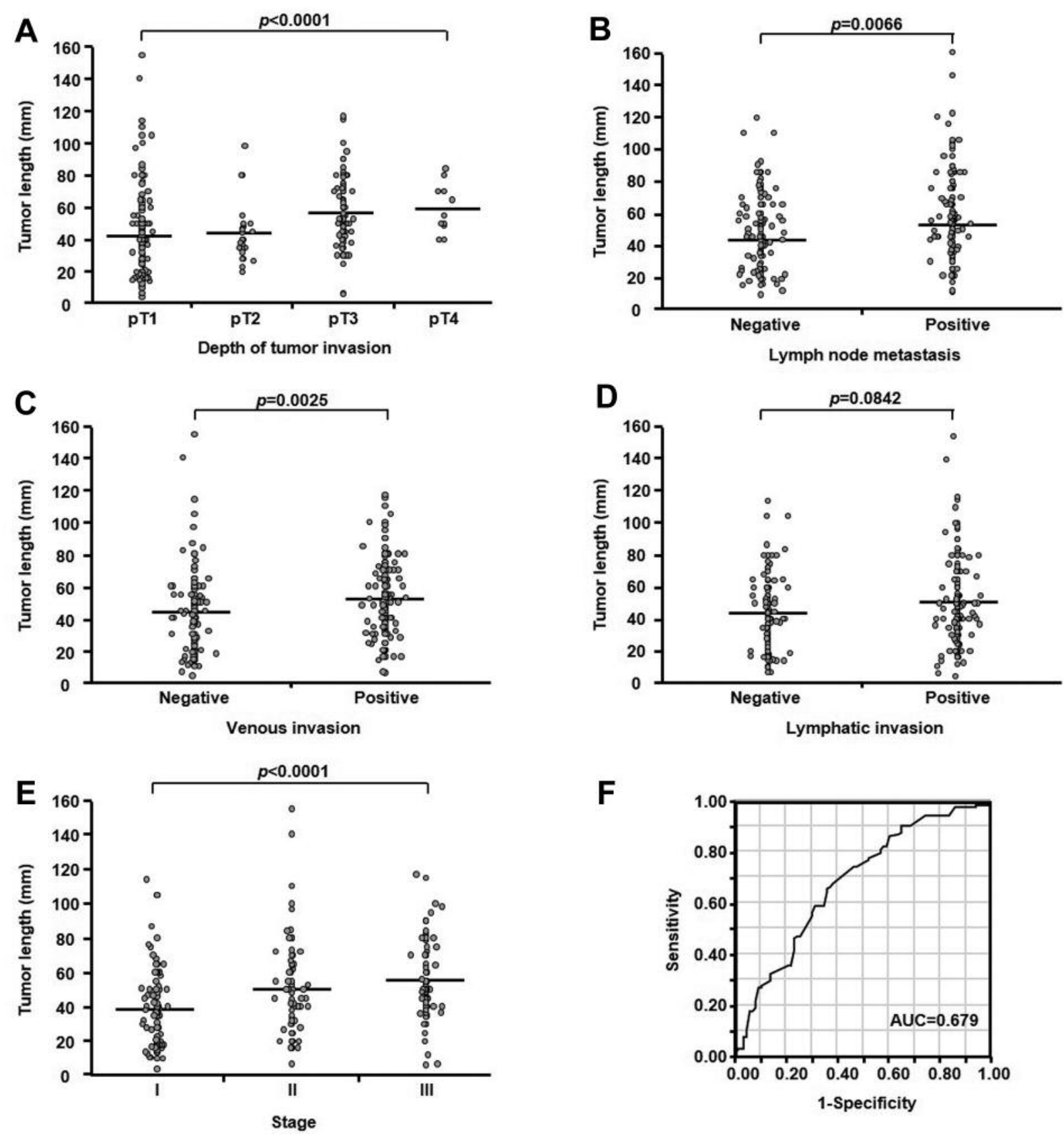

Figure 1. Correlation between tumor length and clinicopathological features in 237 patients with esophageal squamous cell carcinoma. Tumor length was significantly positively associated with depth of tumor invasion (A), lymph node metastasis (B), venous invasion $(C)$, and stage (E), but not with lymphatic invasion (D). Horizontal bars indicate mean tumor length. $F$ : The receiver operating characteristic curve for discriminating patients with early cancer from those with advanced cancer based on tumor length. The area under the curve (AUC) was 0.679.

Prognostic analyses based on PTS. The 5-year survival rate of patients with a PTS of 0,1 , and 2 was $74.1 \%, 65.7 \%$, and $36.6 \%$, respectively $(p<0.0001)$ (Figure 2$)$.

Univariate analysis indicated that lymph node metastasis, venous invasion, lymphatic invasion, and PTS were significantly inversely related to survival $(p<0.0001, p=0.0029$, $p<0.0001$, and $p<0.0001$, respectively) (Table IV). Multivariate analysis selected lymph node metastasis and PTS as being independent prognostic factors $(p=0.0079$ and $p=0.0004$, respectively) (Table IV). 
Table IV. Univariate and multivariate analyses for survival.

\begin{tabular}{|c|c|c|c|c|c|c|c|}
\hline \multirow[b]{2}{*}{ Independent factor } & \multirow[b]{2}{*}{ Comparison } & \multicolumn{3}{|c|}{ Univariate analysis } & \multicolumn{3}{|c|}{ Multivariate analysis } \\
\hline & & Hazard ratio & $95 \%$ CI & $p$-Value & Hazard ratio & $95 \% \mathrm{CI}$ & $p$-Value \\
\hline Lymph node metastasis & Positive $v s$. negative & 3.64 & $2.21-6.24$ & $<0.0001$ & 2.24 & $1.23-4.27$ & 0.0079 \\
\hline Venous invasion & Positive $v s$. negative & 2.05 & $1.27-3.42$ & 0.0029 & 1.22 & $0.74-2.08$ & 0.4478 \\
\hline Lymphatic invasion & Positive $v s$. negative & 3.28 & $1.93-5.90$ & $<0.0001$ & 1.62 & $0.84-3.26$ & 0.1523 \\
\hline Primary tumor score & $1 / 2$ vs. 0 & 3.25 & $2.06-5.18$ & $<0.0001$ & 2.37 & $1.47-3.87$ & 0.0004 \\
\hline
\end{tabular}

\section{Discussion}

In the present study, we propose PTS, determined from the combination of tumor depth and length, as a new prognostic scoring method. We assessed the clinical impact of PTS in 237 patients with ESCC who underwent esophagectomy with lymphadenectomy.

The depth of tumor invasion is well known as the $\mathrm{T}$ factor in TNM classification. In this study, patients with deeper invasion had tumors with increasing burden of lymph node metastasis or advanced stage. Moreover, the log-rank test showed a statistically significant difference in survival between patients with pT1 tumors and patients with pT2, pT3, or pT4 tumors $(p<0.0001)$ (data not shown). These results indicate that the depth of tumor invasion is one of the most important prognostic factors among several kinds of information derived from primary tumor sites.

The clinical significance of tumor length remains unclear in patients with malignancies, including ESCC. Recently, several investigators have demonstrated a close relationship between tumor length and malignant behavior in patients with ESCC (8-12). Zhang et al., in a study of 387 patients with ESCC after surgical resection, reported that tumor length was closely related to the pathological status of tumor depth, lymph node metastasis, and TNM stage ( $p=0.003$, $p=0.024$, and $p=0.019$, respectively). Their study showed that patients with a tumor length $>35 \mathrm{~mm}$ had more aggressive tumors, as indicated by tumor depth, lymph node metastasis, venous invasion, and stage, than those with a tumor length of $35 \mathrm{~mm}$ or less (12). Similarly, we showed in the present study that tumor length was significantly correlated with well-known prognostic factors such as depth of tumor invasion, presence of lymph node metastasis, and stage. Interestingly, ROC analysis demonstrated that tumor length might have clinical utility as a surrogate marker for stratifying patients into those with early and advanced stages of disease. These findings suggest that tumor length, as well as the depth of tumor invasion, are promising factors derived from primary tumor information for predicting tumor progression in patients with ESCC.

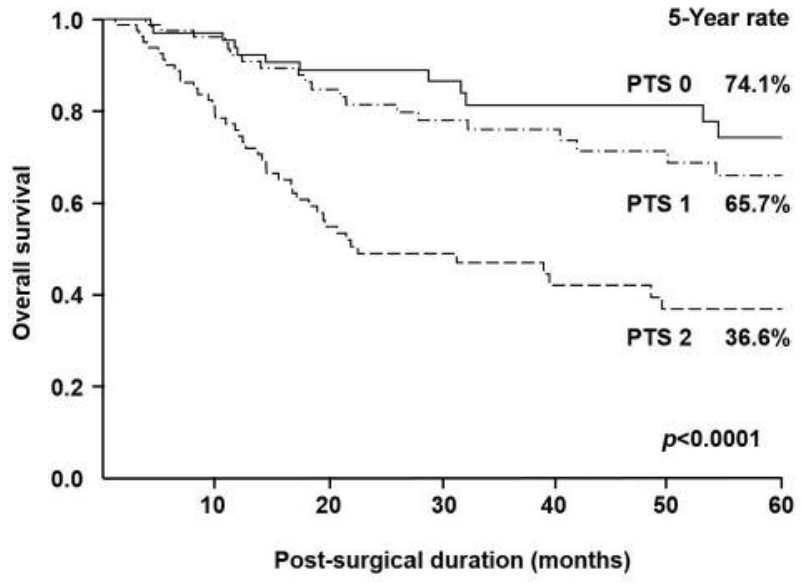

Figure 2. Kaplan-Meier survival curves for patients with esophageal squamous cell carcinoma based on primary tumor score (PTS). The 5 -year survival rates were significantly poorer in patients with higher PTS $(p<0.0001)$.

In a previous study, we reported the clinical significance of PTS calculated from tumor invasion and size in patients with gastric cancer. According to that study, patients with higher PTS were more likely to have lymph node metastasis and advanced-stage cancer than those with lower PTS $(p<0.0001$ for both comparisons) (13). Therefore, we examined the relationship between PTS and clinicopathological factors in patients with ESCC. In the present study, the incidence of lymph node metastasis in patients with a PTS of 2 was higher than that in patients with a PTS of $0(71.6 \% \mathrm{vs} .30 .0 \%$, respectively). The incidence of stage III cancer in patients with a PTS of 2 and 0 was $70.4 \%$ and $4.3 \%$, respectively. These results show that in patients with ESCC, PTS, based on primary tumor information alone, is a useful predictor of tumor progression, simularly to metastatic status of lymph nodes and stage.

Finally, we focused on the prognostic impact of the PTS group in this study. Patients with a higher PTS had a significantly poorer prognosis than those with a lower PTS. 
In particular, the 5-year survival rate of patients with a PTS of 2 was $36.6 \%$. Surprisingly, multivariate analysis demonstrated that PTS was one of the most important prognostic factors. Accordingly, the PTS may be a simple tool for predicting disease recurrence and long-term outcome in patients with ESCC. Our hypothesis suggests that PTS as well as conventional TNM stage may support the clinical selection of patients needing adjuvant chemotherapy in the postoperative management of ESCC.

The present study had several limitations. This preliminary study was a retrospective analysis with a small sample size conducted at a single institution. The median follow-up period was short (26 months). These limitations may have affected several results. Larger validation studies are needed to strengthen our results obtained in patients with ESCC.

In conclusion, we demonstrated that PTS, determined by tumor depth and length, had a clinical utility as a predictor of tumor progression and prognosis in patients with ESCC. The PTS system may be a useful tool for planning therapeutic strategies to prevent disease recurrence in postoperative patients with ESCC.

\section{Conflicts of Interest}

The Authors have no conflicts of interest or financial ties to disclose.

\section{References}

1 Lin Y, Totsuka Y, He Y, Kikuchi S, Qiao Y, Ueda J, Wei W, Inoue $\mathrm{M}$ and Tanaka $\mathrm{H}$ : Epidemiology of esophageal cancer in Japan and China. J Epidemiol 23: 233-242, 2013.

2 Ando N, Ozawa S, Kitagawa Y, Shinozawa Y and Kitajima M: Improvement in the results of surgical treatment of advanced squamous esophageal carcinoma during 15 consecutive years. Ann Surg 232: 225-232, 2000.

3 Akutsu Y, Uesato M, Shuto K, Kono T, Hoshino I, Horibe D, Sazuka T, Takeshita N, Maruyama T, Isozaki Y, Akanuma N and Matsubara $\mathrm{H}$ : The overall prevalence of metastasis in $\mathrm{T} 1$ esophageal squamous cell carcinoma: A retrospective analysis of 295 patients. Ann Surg 257: 1032-1038, 2013.

4 Japanese Gastric Cancer Association: Japanese Gastric Cancer Treatment Guidelines 2014 (Ver. 4). Gastric Cancer 20: 1-19, 2017.
5 Takahashi K, Miyashita M, Nomura T, Makino H, Futami R, Kashiwabara M, Katsuta M and Tajiri T: Serum p53 antibody as a predictor of early recurrence in patients with postoperative esophageal squamous cell carcinoma. Dis Esophagus 20: 117122, 2007.

6 Amin MB, Edge S, Greene F, Byrd DR, Brookland RK, Washington MK, Gershenwald JE, Compton CC, Hess KR, Sullivan DC, Jessup JM, Brierley JD, Gaspar LE, Schilsky RL, Balch CM, Winchester DP, Asare EA, Madera M, Gress DM and Meyer LR (eds): AJCC Cancer Staging Manual, eighth edition. New York, Springer, pp. 185-202, 2017.

7 Japan Esophageal Society: Japanese Classification of Esophageal Cancer, 11th Edition. Esophagus 14: 1-65, 2017.

8 Eloubeidi MA, Desmond R, Arguedas MR, Reed CE and Wilcox CM: Prognostic factors for the survival of patients with esophageal carcinoma in the U.S.: The importance of tumor length and lymph node status. Cancer 95: 1434-1443, 2002.

9 Griffiths EA, Brummell Z, Gorthi G, Pritchard SA and Welch IM: Tumor length as a prognostic factor in esophageal malignancy: univariate and multivariate survival analyses. J Surg Oncol 93: 258-267, 2006.

10 Yendamuri S, Swisher SG, Correa AM, Hofstetter W, Ajani JA, Francis A, Maru D, Mehran RJ, Rice DC, Roth JA, Walsh GL and Vaporciyan AA: Esophageal tumor length is independently associated with long-term survival. Cancer 115: 508-516, 2009.

11 Wang BY, Goan YG, Hsu PK, Hsu WH and Wu YC: Tumor length as a prognostic factor in esophageal squamous cell carcinoma. Ann Thorac Surg 91: 887-893, 2011.

12 Zhang H, Tang P, Miao X, Gao Y, Shang X, Gong L, Ma Z, Yang M, Jiang H, Zhan Z, Meng B and Yu Z: Does tumor size improve the accuracy of prognostic prediction in patients with esophageal squamous cell carcinoma after surgical resection? Oncotarget 7: 66623-66634, 2016.

13 Haraguchi N, Arigami T, Uenosono Y, Yanagita S, Uchikado Y, Mori S, Kurahara H, Kijima Y, Nakajo A, Maemura K, Ishigami $\mathrm{S}$ and Natsugoe S: Clinical significance of primary tumor score determined by tumor depth and size in patients with resectable gastric cancer. Oncotarget 9: 8512-8520, 2018. 